\title{
Methylation of Promoter Regions of Genes of the Human Intrauterine Renin Angiotensin System and Their Expression
}

\author{
Shane D. Sykes, ${ }^{1,2}$ Carolyn Mitchell, ${ }^{2,3}$ Kirsty G. Pringle, ${ }^{1,2}$ Yu Wang, \\ Tamas Zakar, ${ }^{2,3,4}$ and Eugenie R. Lumbers ${ }^{1,2}$ \\ ${ }^{1}$ School of Biomedical Sciences and Pharmacy, University of Newcastle, Newcastle, NSW 2308, Australia \\ ${ }^{2}$ Mothers and Babies Research Centre, Hunter Medical Research Institute, Newcastle, NSW 2308, Australia \\ ${ }^{3}$ School of Medicine and Public Health, University of Newcastle, Newcastle, NSW 2308, Australia \\ ${ }^{4}$ Department of Obstetrics and Gynaecology, John Hunter Hospital, Newcastle, NSW 2308, Australia
}

Correspondence should be addressed to Eugenie R. Lumbers; eugenielumbers@gmail.com

Received 19 January 2015; Revised 11 March 2015; Accepted 16 March 2015

Academic Editor: Małgorzata Kotula-Balak

Copyright (C) 2015 Shane D. Sykes et al. This is an open access article distributed under the Creative Commons Attribution License, which permits unrestricted use, distribution, and reproduction in any medium, provided the original work is properly cited.

\begin{abstract}
The intrauterine renin angiotensin system (RAS) is implicated in placentation and labour onset. Here we investigate whether promoter methylation of RAS genes changes with gestation or labour and if it affects gene expression. Early gestation amnion and placenta were studied, as were term amnion, decidua, and placenta collected before labour (at elective caesarean section) or after spontaneous labour and delivery. The expression and degree of methylation of the prorenin receptor (ATP6AP2), angiotensin converting enzyme (ACE), angiotensin II type 1 receptor (AGTR1), and two proteases that can activate prorenin (kallikrein, KLK1, and cathepsin D, CTSD) were measured by qPCR and a DNA methylation array. There was no effect of gestation or labour on the methylation of RAS genes and CTSD. Amnion and decidua displayed strong correlations between the percent hypermethylation of RAS genes and CTSD, suggestive of global methylation. There were no correlations between the degree of methylation and mRNA abundance of any genes studied. KLK1 was the most methylated gene and the proportion of hypermethylated KLK1 alleles was lower in placenta than decidua. The presence of intermediate methylated alleles of $K L K 1$ in early gestation placenta and in amnion after labour suggests that KLK1 methylation is uniquely dynamic in these tissues.
\end{abstract}

\section{Introduction}

Human intrauterine tissues (amnion, chorion, decidua, placenta, and myometrium) all express genes that affect the rate of production and actions of angiotensin peptides $[1,2]$. Prorenin (the inactive form of renin) is secreted mainly by decidua. It requires proteolysis or binding to the prorenin receptor (ATP6AP2) to become active [3].

There are two potential pathways via which the decidual and amniotic renin angiotensin systems (RASs) could be involved in parturition. First, during labour, there is a marked up regulation of inflammatory genes in term amnion, decidua, and placenta, the so-called "inflammatory signature" [4]. Angiotensin II (Ang II), the major product of the renin angiotensin cascade formed from Ang I by angiotensin converting enzyme $(A C E)$ and acting through the angiotensin II type 1 receptor (AGTR1), is a proinflammatory peptide [5]. Second, renin and prorenin acting independent of Ang II stimulate prostaglandin endoperoxide synthase-2 (PGHS-2) production in primary amnion cells in vitro [6]. Both kallikrein and cathepsin D can proteolytically activate prorenin and are present in human intrauterine tissues [710]. Cathepsin D is a lysosomal enzyme that can also form angiotensin I from angiotensinogen. It has been implicated in promoting invasion by endometriotic tissue [8]. Kallikrein, a serine protease, is the key enzyme involved in activation of the kallikrein-kinin system (KKS). The KKS has actions that generally oppose those of the RAS in regulating blood pressure; however, in some circumstances the KKS has effects that are similar to the RAS in regulating vascular function, inflammation, and cell growth [11].

Cell specific DNA methylation contributes to cell identity. Although CpG methylation status is conferred early in development [12-14], it can change [15-17] and could 
be one pathway regulating intrauterine gene expression throughout gestation. Methylation of $\mathrm{CpG}$ dinucleotides in the promoter region of a gene represses transcription either by inhibiting binding of transcription factors or by binding proteins that condense the chromatin structure. Three RAS genes essential for the generation or activity of Ang II in human intrauterine tissues, namely, the prorenin receptor (ATP6AP2), angiotensin converting enzyme (ACE), and the angiotensin II type 1 receptor (AGTR1) have a high density of $\mathrm{CpG}$ motifs in their promoter region, as do kallikrein (KLK1) and cathepsin D (CTSD). We have previously demonstrated that placental expression of ATP6AP2 and AGTR1 were highest in early gestation while the expression of $A C E$ was greatest at term [1]. We attributed this difference in $A C E$ mRNA abundance to the increased density of fetal endothelial cells which are the only placental cells containing ACE [1]. We postulated that if the methylation status of ATP6AP2 and AGTR1 influenced their expression then the level of methylation of these two genes would also increase throughout gestation.

The methylation of RAS genes within the intrauterine tissues could also be altered during labour and affect their expression, since decidual ATP6AP2 and amnion ACE expressions are both downregulated in labour $[2,18]$. We therefore determined the methylation status of ATP6AP2, $A C E$, and $A G T R 1$ in these tissues across gestation and during labour and correlated this with changes in their expression. In addition, we explored methylation-dependent regulation further by determining for the first time the effects of gestation and labour on expression of KLKI and CTSD in gestational tissues and correlating expression levels with the methylation density of the respective gene promoters.

\section{Methods}

2.1. Ethics Approval. The Hunter Area Research Ethics Committee and the University of Newcastle Research Ethics Committee have given approval to carry out this work.

2.2. Tissues. Early gestation placenta $(n=4)$ and amnion $(n=8)$ were collected after informed consent from women undergoing elective termination of pregnancy (10-18 weeks of gestation). Term tissues (37-41 weeks of gestation) were collected from women presenting to the John Hunter Hospital (Newcastle, Australia) with uncomplicated singleton pregnancies following informed consent. Term amnion $(n=$ $8)$, decidua $(n=8)$, and placenta $(n=4)$ were obtained after elective caesarean section in the absence of labour. Amnion and decidua were also collected from women after spontaneous labour and vaginal delivery and processed within 30 min of collection $(n=8)$.

Women were excluded if there was a history of treatment with nonsteroidal anti-inflammatory drugs, infection, chorioamnionitis, asthma, or induced labour. Amnion and decidua were collected from the reflected membranes and dissected at least $1 \mathrm{~cm}$ away from the adjacent placenta; amnion was then subsequently peeled from the choriodecidua and the decidua isolated from the chorion laevae by sharp dissection as described previously [19]. Placental tissue was excised from several sites across the placental bed. Samples used in this study were from a tissue bank of previously collected tissues and thus were not matched by patients. All tissues were snap-frozen in liquid nitrogen for storage at $-80^{\circ} \mathrm{C}$ until further processing.

2.3. Methylation Density Analyses. All samples were analysed using a customized Methyl-Profiler DNA Methylation PCR Array System (SA Biosciences, Doncaster, Vic, Australia, distributed by Qiagen as EpiTect Methyl qPCR System) according to the manufacturer's specifications. Briefly, genomic DNA was isolated from 20-25 mg of crushed frozen placenta, amnion, or decidua using the QIAamp DNA mini kit (Qiagen, Chadstone, Vic, Australia) according to the manufacturer's instructions. Genomic DNA was then treated with one of 4 enzyme treatments provided in the DNA Methylation Enzyme Kit (SA Biosciences). Restriction digests using either the methylation-dependent or the methylationsensitive enzyme contained $1 \mu \mathrm{L}$ of enzyme solution, while digests containing both enzymes contained $1 \mu \mathrm{L}$ of each. Mock digests contained no restriction enzyme. Samples were made to a final volume of $30 \mu \mathrm{L}$ containing $125 \mathrm{ng}$ of isolated genomic DNA in digestion buffer. The four digested DNA solutions were processed for PCR analysis by adding $\mathrm{RT}^{2}$ SYBR Green qPCR Master Mix (SA Biosciences) and water to a final concentration of $7.8 \mathrm{ng}$ of DNA per well. The PCR plates were supplied with the appropriate primers for ACE, AGTR1, ATP6AP2, CTSD, and KLK1 genes coated to the wells of each plate. The PCRs were performed on an Applied Biosystems 7500 Real-Time PCR System (Applied Biosystems, Mulgrave, Vic, Australia) according to the manufacturer's instructions. The primer sequences and amplicon positions have been optimized by the manufacturer and are considered proprietary. All amplicons included the transcription start site (TSS), several hundred base pairs upstream and downstream of the TSS, and at least one CpG island including or near to the TSS. The methylation status of the genes was calculated using software supplied with the DNA MethylProfiler PCR Array (SA Biosciences) and according to the manufacturer's recommendations. This software reports the proportions of gene alleles within each DNA sample that are hypermethylated, intermediately methylated, or hypomethylated. This technique relies on the ability of the methylationdependent restriction enzyme (which cleaves methylated DNA) and methylation-sensitive restriction enzyme (which cleaves only at unmethylated recognition sites) to digest the DNA differentially. Assays were reported as failures if delta$\mathrm{Ct}$ values between digests with both restriction enzymes or the mock-digest with neither enzyme were $\leq 2$, representing a sample where $\geq 25 \%$ of the template was digestion-resistant. The principle and validation of this DNA methylation density monitoring system have been published previously [20, 21].

2.4. Quantitative Real-Time RT-PCR ( $q P C R)$. Total RNA was isolated from $0.2 \mathrm{~g}$ of tissue using TRIzol reagent (Invitrogen, Mulgrave, Vic, Australia) according to the manufacturer's instructions. Samples were purified and contaminating DNA were removed by treatment with DNase I on RNeasy Mini kit spin columns (Qiagen) and quantification and purity of 
RNA were determined using spectrophotometry (NanoDrop ND-1000, NanoDrop Technologies, Rockland, DE, USA). Reverse transcription was carried out with Superscript III RTkit using random hexamer primers (Invitrogen). Quantitative real-time PCR was conducted with SYBR Green for detection using an Applied Biosystems 7500 Real-Time PCR System. Each reaction contained $5 \mu \mathrm{L}$ SYBR Green, cDNA generated from $10 \mathrm{ng}$ of total RNA, primers, and water, made up to a final volume of $10 \mu \mathrm{L}$. Primers for $K L K 1$ were designed as previously described [22], while all other primers except CTSD have been described elsewhere $[2,18]$. The primers for CTSD were designed with Primer Express 3.0 software (Applied Biosystems) using NCBI reference sequence NM_001909.3. The sequences of the forward and reverse primers for detecting CTSD mRNA were 5'CCTGAGCAGGGACCCAGAT and $5^{\prime}$ GGTGACATTCAGGTAGGACAGAGA, respectively. This set of primers was used at the optimized concentration of $100 \mathrm{nM}$. All samples were assayed in triplicate using the following reaction conditions: incubation at $50^{\circ} \mathrm{C}$ for 2 minutes, incubation at $95^{\circ} \mathrm{C}$ for 10 minutes, 40 cycles of 15 seconds at $95^{\circ} \mathrm{C}$, and 40 cycles of 1 minute at $60^{\circ} \mathrm{C}$. Dissociation curves were established after all qPCR amplifications to ascertain the homogeneity of the products. Data from qPCR were analysed according to the $2^{-\Delta \Delta \mathrm{Ct}}$ method using $\beta$-actin mRNA as an internal reference and efficiency ratios were verified to be 2 for all genes [23]. The abundance of $\beta$-actin mRNA was determined to be equal in all gestational and tissue groups except for amnion, in which $\beta$-actin mRNA levels were $14 \%$ less in early gestation than those of the other amnion groups. Therefore, the relative mRNA abundance measured in early gestation amnion samples was multiplied by 1.14 in order to compensate for this difference in the average reference mRNA level. Term placental cDNA was used as a calibrator for all genes except $K L K 1$, where cDNA from SW48 cells (a colorectal cancer cell line) was used as the calibrator instead.

2.5. Statistics and Data Analysis. Data were analysed using Mann-Whitney $U$ tests, Kruskal-Wallis tests with post hoc Dunn's multiple comparison tests, and Spearman correlations. Significance was accepted at the 5\% level. Data are presented as Tukey box plots with outliers shown as filled in circles. To determine whether or not any individual gene had a different degree of hypermethylation of alleles from levels measured in the 5 genes measured in this study and the 7 genes studied by [24], significant relationships identified using Spearman correlations are visually represented by scatter plots with logarithmic scales. GraphPad Prism 6.0 (GraphPad Software Inc.) was used for statistical analyses and preparation of figures.

\section{Results}

3.1. The Effects of Gestational Age and Labour on RAS, CTSD, and KLK1 Gene Methylation and mRNA Expression in Amnion. In the amnion, the proportion of hypermethylated ACE, AGTR1, ATP6AP2, and CTSD gene alleles varied among individuals but showed no significant differences with gestational age or labour (Figure 1). One early gestation amnion had intermediate methylation in the ATP6AP2 gene, and there was no intermediate methylation of ACE, AGTR1, and CTSD genes in any amnion sample. A significantly greater proportion of $K L K 1$ alleles was hypermethylated in early gestation amnion compared to amnions collected after spontaneous labour and vaginal delivery at term $(P<$ 0.02, Figure 1). Furthermore, intermediate KLK1 methylation density was found in one caesarean derived amnion (hypermethylated, 25.1\%; intermediately methylated, 30.6\%; and hypomethylated, $44.3 \%$ ) and 2 amnion samples collected after labour (8.8 and 2.1\% hypermethylated; 83.3 and $96.0 \%$ intermediately methylated; and 7.9 and $1.9 \%$ hypomethylated, resp.).

Amnion ACE, AGTR1, and KLKI mRNA levels did not change with gestation, but there was a significant increase in ATP6AP2 and CTSD mRNA abundance in late pregnancy after both caesarean and vaginal delivery (for ATP6AP2, $P<$ 0.001 and $P<0.05$; for CTSD, $P=0.001$ and $P<0.01$, resp.). Labour had no effect on the expression of any of these amniotic genes, and we found no correlation between mRNA abundance and the proportion of hypermethylated alleles.

3.2. The Effects of Labour on RAS, CTSD, and KLK1 Gene Methylation and $m R N A$ Expression in Decidua. There was no effect of labour on the proportion of RAS gene alleles that were hypermethylated (Figure 2) in the decidua. The mRNA abundances of the RAS genes were also unchanged, except for AGTR1, which was lower in women who had a spontaneous labour and vaginal delivery than in those who delivered by caesarean section in the absence of labour (Figure 2). In the decidua the mRNA abundances of RAS genes, CTSD, and $K L K 1$ were not correlated with the proportion of hypermethylated alleles.

\subsection{The Effects of Gestational Age on RAS, CTSD, and KLK1} Gene Methylation and mRNA Expression in Placenta. The proportion of RAS gene alleles that were hypermethylated in the placenta was not affected by gestational age (data not shown). In early gestation placentae, however, several genes showed a high level of intermediate methylation (Table 1). No late gestation placentae showed intermediate methylation; all studied genes were hypomethylated. The small number of samples with intermediate DNA methylation did not allow statistical analyses to be conducted between gestational age groups.

There were no gestational changes in ACE, AGTR1, and KLKI mRNA levels, but ATP6AP2 mRNA abundance decreased and CTSD mRNA abundance increased with gestational age (Figure 3). We found no correlations in the placenta between mRNA abundance and the proportion of hypermethylated alleles of any gene studied.

3.4. Effects of Gestational Tissue Type on the Proportion of Methylated RAS, CTSD, and KLK1 Alleles and mRNA Expression. We compared levels of RAS gene methylation in amnion, decidua, and placenta obtained at elective caesarean section (Figure 4). Only KLK1 showed tissue specific differential methylation with decidua exhibiting a greater proportion 

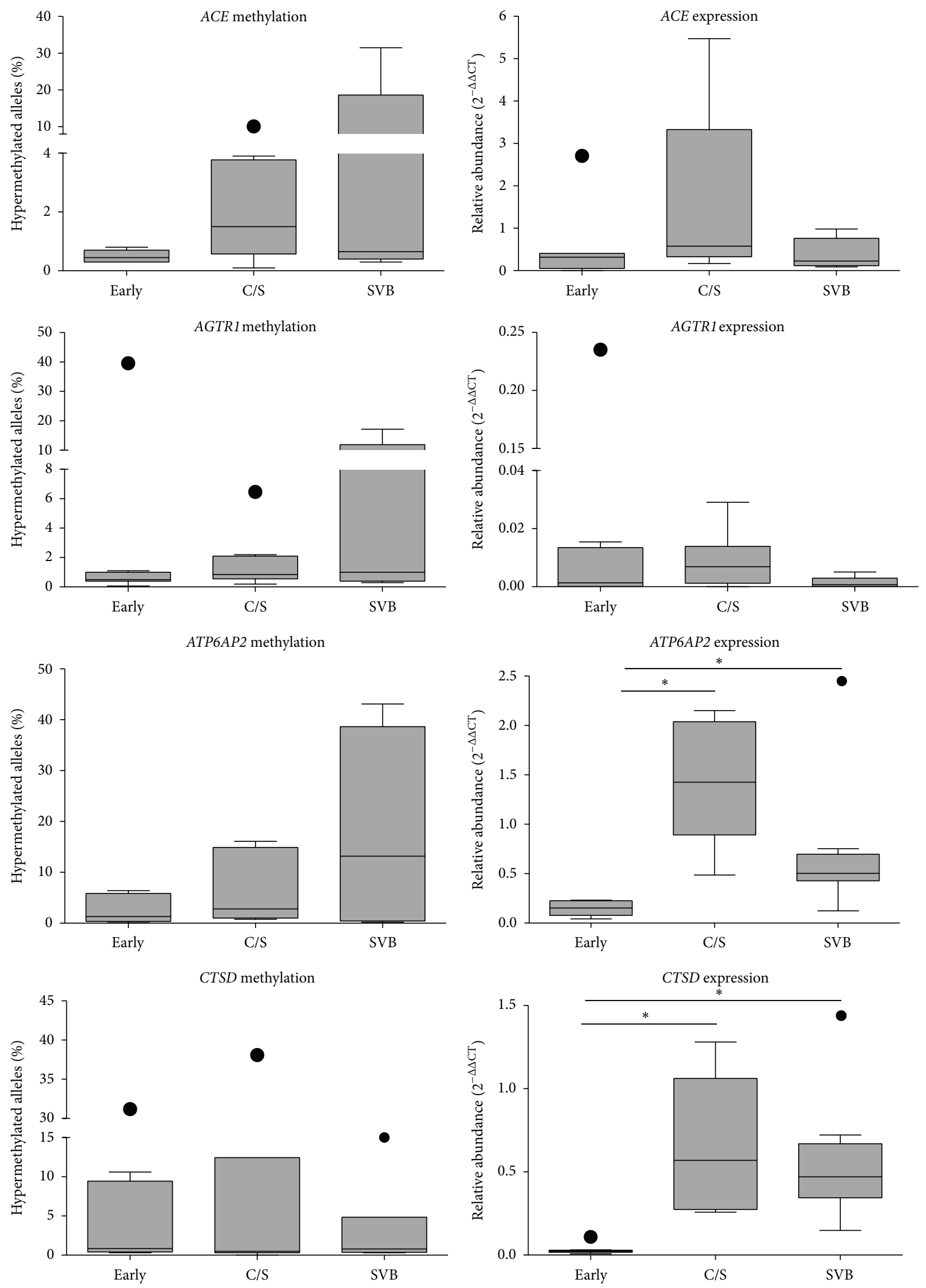

Figure 1: Continued. 

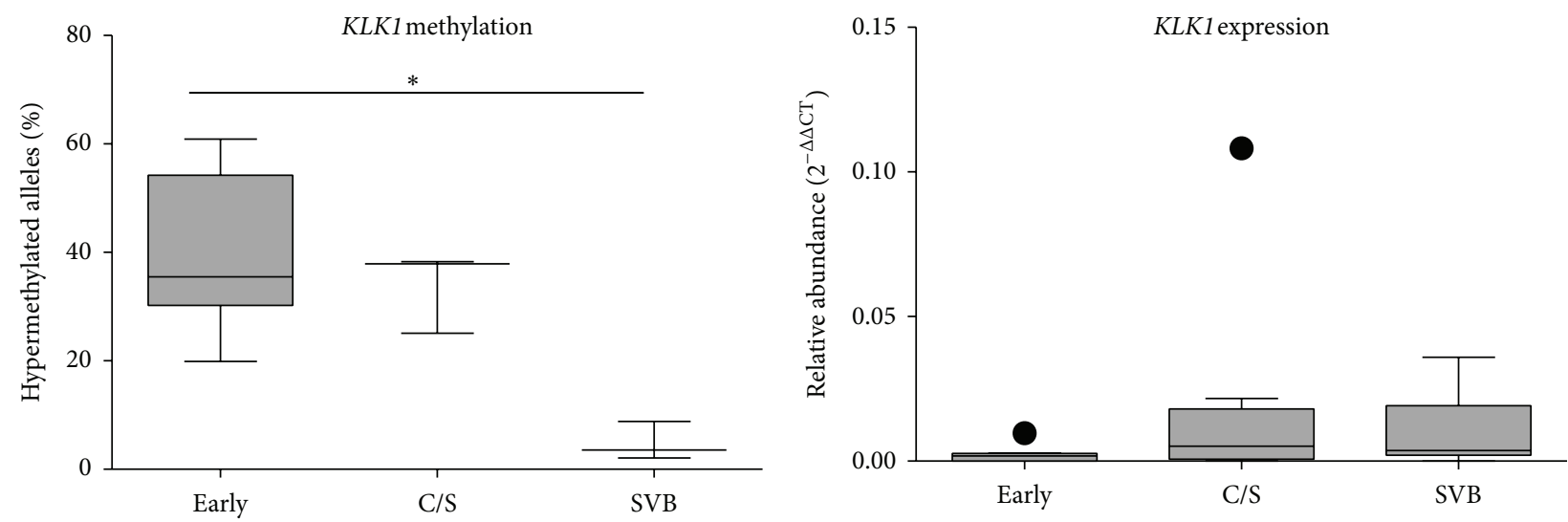

FIGURE 1: The proportions of hypermethylated gene alleles for ACE, AGTR1, ATP6AP2, CTSD, and KLK1 and their corresponding mRNA abundances in amnion collected from early gestation (early, $n=8$ ) and normal term pregnancies either by elective caesarean section prior to the onset of labour (C/S, $n=8)$ or after labour in women who had a spontaneous labour and vaginal delivery (SVB, $n=8)$. Significant differences were determined with Kruskal-Wallis tests using Dunn's multiple comparison test, with significant differences denoted by $*$ (adjusted $P<0.05$ ). Data are presented as Tukey box plots with outliers shown as filled in circles. DNA methylation profiles could not be measured in early gestation for $1 / 8 A C E$ and $K L K 1$ samples, from C/S for $1 / 8$ ATP6AP2, 2/8 CTSD, and 5/8 KLK1 samples, and from SVB for 1/8 AGTR1, 4/8 ATP6AP2, 2/8 CTSD, and 5/8 KLK1 samples.
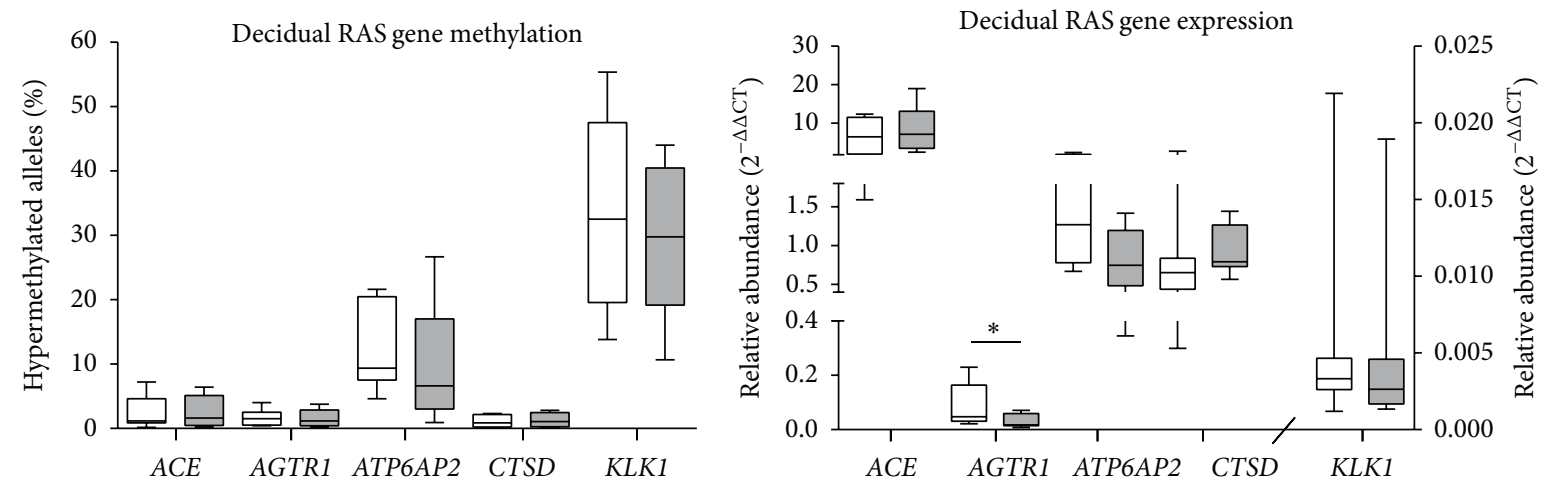

FIGURE 2: The proportions of hypermethylated alleles of ACE, AGTR1, ATP6AP2, CTSD, and KLK1 and the mRNA abundances of these genes isolated from decidua collected from normal term pregnancies either by elective caesarean section prior to the onset of labour (white bars, $n=8$ ) or from women who had spontaneous labour and a vaginal delivery (grey bars, $n=8$ ). Significant differences were determined using Mann-Whitney $U$ tests, with significant differences denoted by $*(P<0.05)$. Data are presented as Tukey box plots. DNA methylation profiles could not be detected in the absence of labour for 1/8 ACE, 1/8 AGTR1, 2/8 ATP6AP2, and 3/8 KLK1 samples and from after labour for 3/8 KLK1 samples.
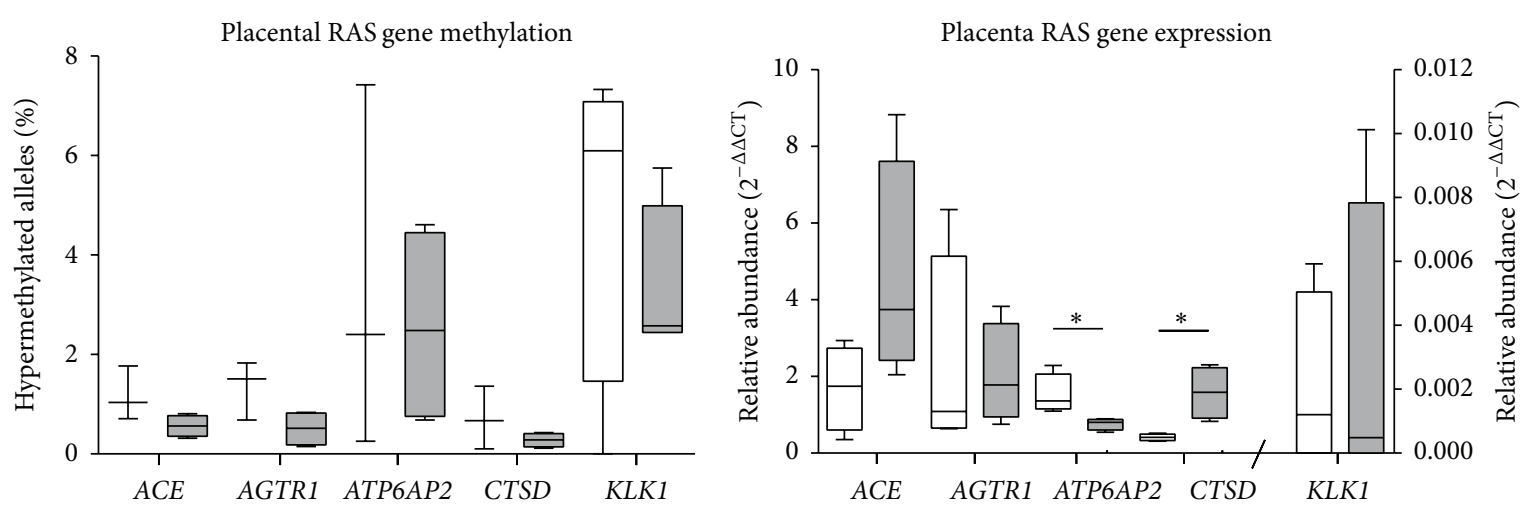

Figure 3: The mRNA abundance of ACE, AGTR1, ATP6AP2, CTSD, and KLK1 in early and late gestation placentae $(n=4$, resp.). Significant differences were determined using Mann-Whitney $U$ tests, with significant differences denoted by $*(P<0.05)$. Data are presented as Tukey box plots. 

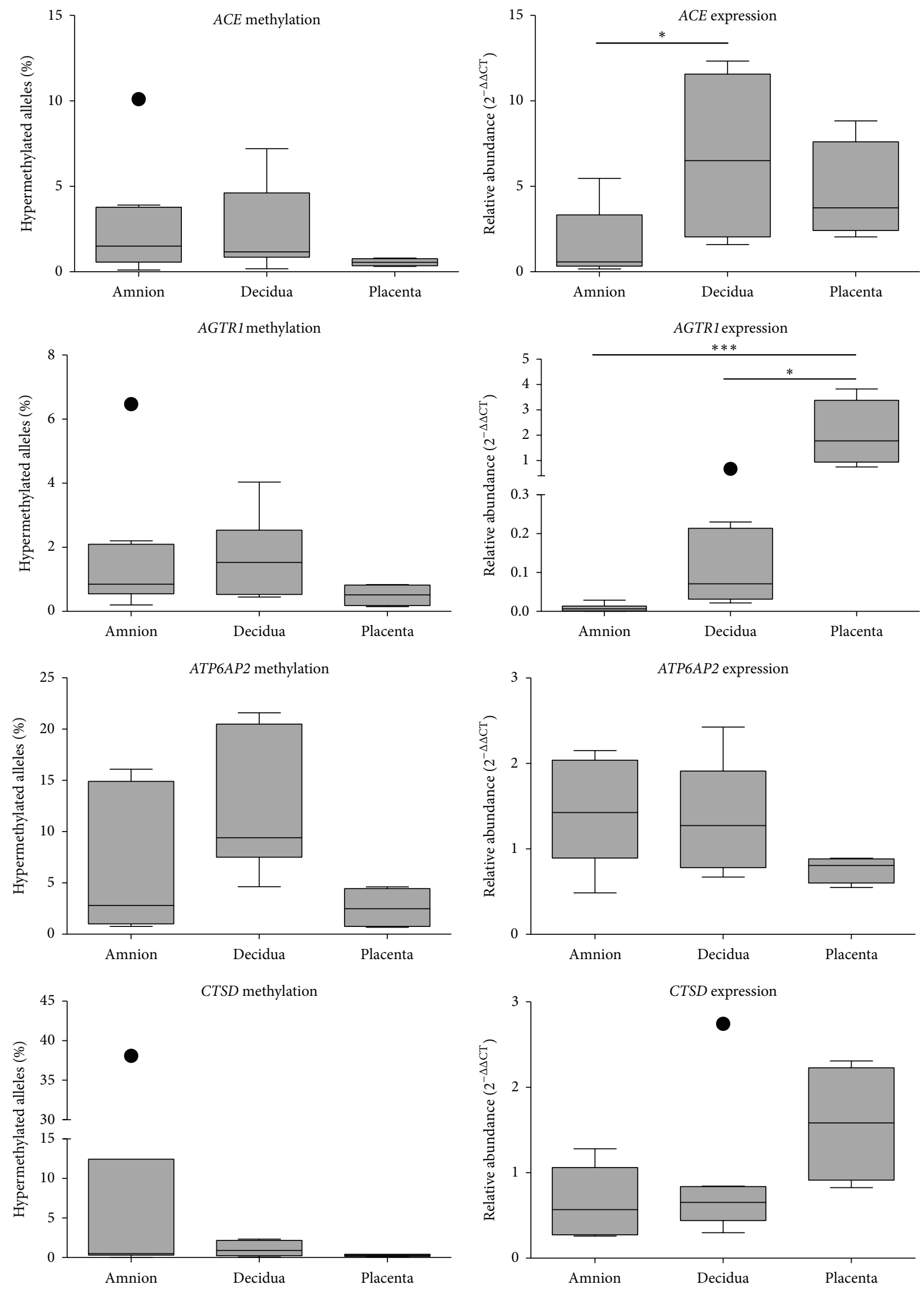

FIgURE 4: Continued. 

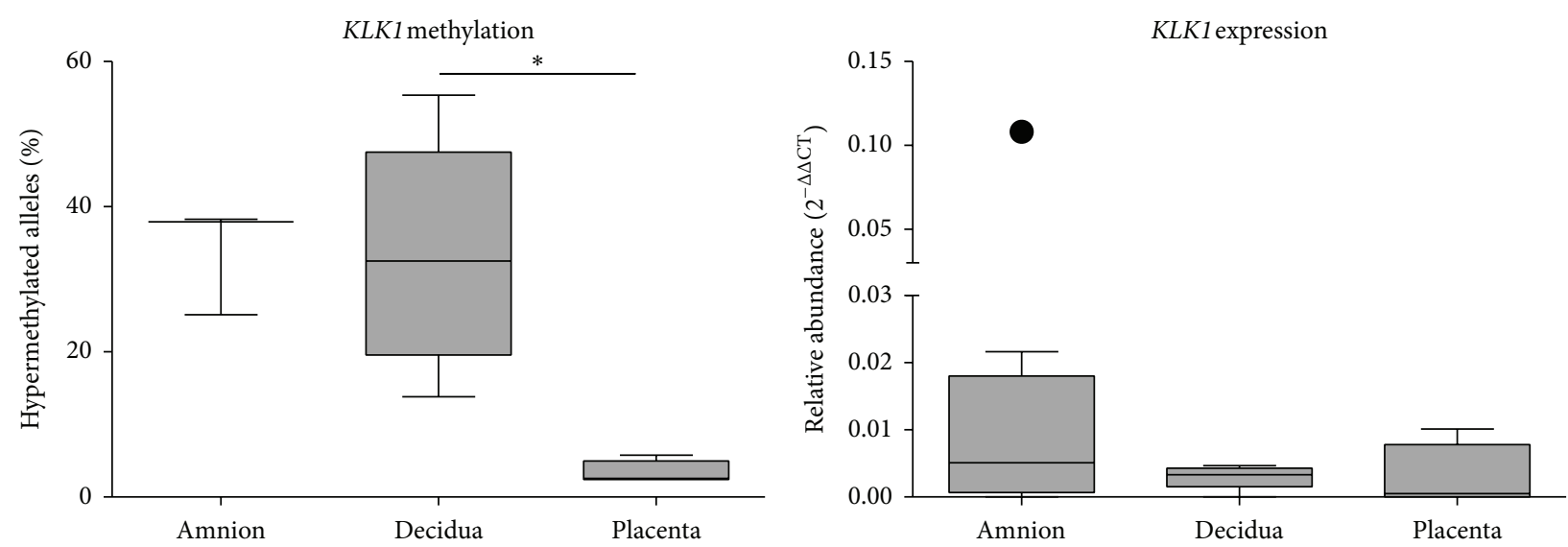

FIGURE 4: The proportions of hypermethylation in alleles of ACE, AGTR1, ATP6AP2, CTSD, and KLK1 and the mRNA abundance of these genes within amnion, decidua, and placenta collected from term pregnancies before the onset of labour. Significant differences were determined with Kruskal-Wallis tests using Dunn's multiple comparison test, with significant differences to amnion denoted by $*(P<0.05)$ and $* * *(P<0.001)$. Data are presented as Tukey box plots with outliers displayed as filled circles.

TABLE 1: The percentage of gene alleles that were hypermethylated, intermediately methylated, and hypomethylated for ACE, AGTR1, $A T P 6 A P 2, C T S D$, and KLK1 in placenta from women in early gestation $(n=4)$ or at delivery in late gestation in the absence of labour $(n$ $=4, \mathrm{C} / \mathrm{S})$.

\begin{tabular}{|c|c|c|c|c|c|c|c|}
\hline \multirow{2}{*}{ Gene } & \multirow{2}{*}{ Sample } & \multicolumn{3}{|c|}{ Early gestation } & \multicolumn{3}{|c|}{$\mathrm{C} / \mathrm{S}$} \\
\hline & & \% Hypermethyl. & \% Inter. methyl. & \% Hypomethyl. & \% Hypermethyl. & $\%$ Inter. methyl. & \% Hypomethyl. \\
\hline \multirow{4}{*}{$A C E$} & 1 & 1.0 & 0.0 & 99.0 & 0.3 & 0.0 & 99.7 \\
\hline & 2 & - & - & - & 0.8 & 0.0 & 99.2 \\
\hline & 3 & 1.8 & 0.0 & 98.2 & 0.5 & 0.0 & 99.5 \\
\hline & 4 & 0.7 & 0.0 & 99.3 & 0.7 & 0.0 & 99.3 \\
\hline \multirow{4}{*}{ AGTR1 } & 1 & 1.8 & 0.0 & 98.2 & 0.3 & 0.0 & 99.7 \\
\hline & 2 & - & - & - & 0.8 & 0.0 & 99.2 \\
\hline & 3 & 1.5 & 0.0 & 98.5 & 0.2 & 0.0 & 99.8 \\
\hline & 4 & 0.7 & 99.3 & 0.0 & 0.8 & 0.0 & 99.2 \\
\hline \multirow{4}{*}{ ATP6AP2 } & 1 & 2.4 & 0.0 & 97.6 & 4.6 & 0.0 & 95.4 \\
\hline & 2 & 7.4 & 92.4 & 0.2 & 1.0 & 0.0 & 99.0 \\
\hline & 3 & 0.3 & 78.0 & 21.7 & 4.0 & 0.0 & 96.0 \\
\hline & 4 & - & - & - & 0.7 & 0.0 & 99.3 \\
\hline \multirow{4}{*}{ CTSD } & 1 & 0.7 & 0.0 & 99.3 & 0.1 & 0.0 & 99.9 \\
\hline & 2 & - & - & - & 0.3 & 0.0 & 99.7 \\
\hline & 3 & 1.4 & 0.0 & 98.6 & 0.4 & 0.0 & 99.6 \\
\hline & 4 & 0.1 & 99.8 & 0.1 & 0.2 & 0.0 & 99.8 \\
\hline \multirow{4}{*}{$K L K 1$} & 1 & 5.8 & 0.0 & 94.2 & 2.4 & 0.0 & 97.6 \\
\hline & 2 & 6.4 & 93.6 & 0.0 & 5.8 & 0.0 & 94.2 \\
\hline & 3 & 7.3 & 0.0 & 92.7 & 2.7 & 0.0 & 97.3 \\
\hline & 4 & 0.0 & 100.0 & 0.0 & 2.5 & 0.0 & 97.5 \\
\hline
\end{tabular}

Inter. $=$ intermediate, methyl. $=$ methylation. Samples with no value $(-)$ had a DNA template in which $\geq 25 \%$ was digestion-resistant from combined methylation sensitive and resistant enzymes.

of hypermethylated $K L K 1$ alleles than placenta $(P<0.05)$. No differences were seen in KLK1 mRNA abundance between these tissues.

Furthermore, ACE mRNA abundance was greater in decidua than in amnion $(P<0.05)$ and placental AGTR1 mRNA abundance was higher than that measured in both decidua and amnion $(P<0.05$ and $P<0.001$, resp.).
3.5. Relationships between the Proportions of Methylated RAS, CTSD, and KLK1 Alleles in the Gestational Tissue. In amnion and decidua, the proportions of hypermethylated ACE, AGTR1, and ATP6AP2 alleles were positively correlated (Figure 5). Furthermore, the proportions of hypermethylated CTSD alleles were correlated with the proportions of hypermethylated ACE and AGTR1 alleles (Figure 6) in both tissues. 

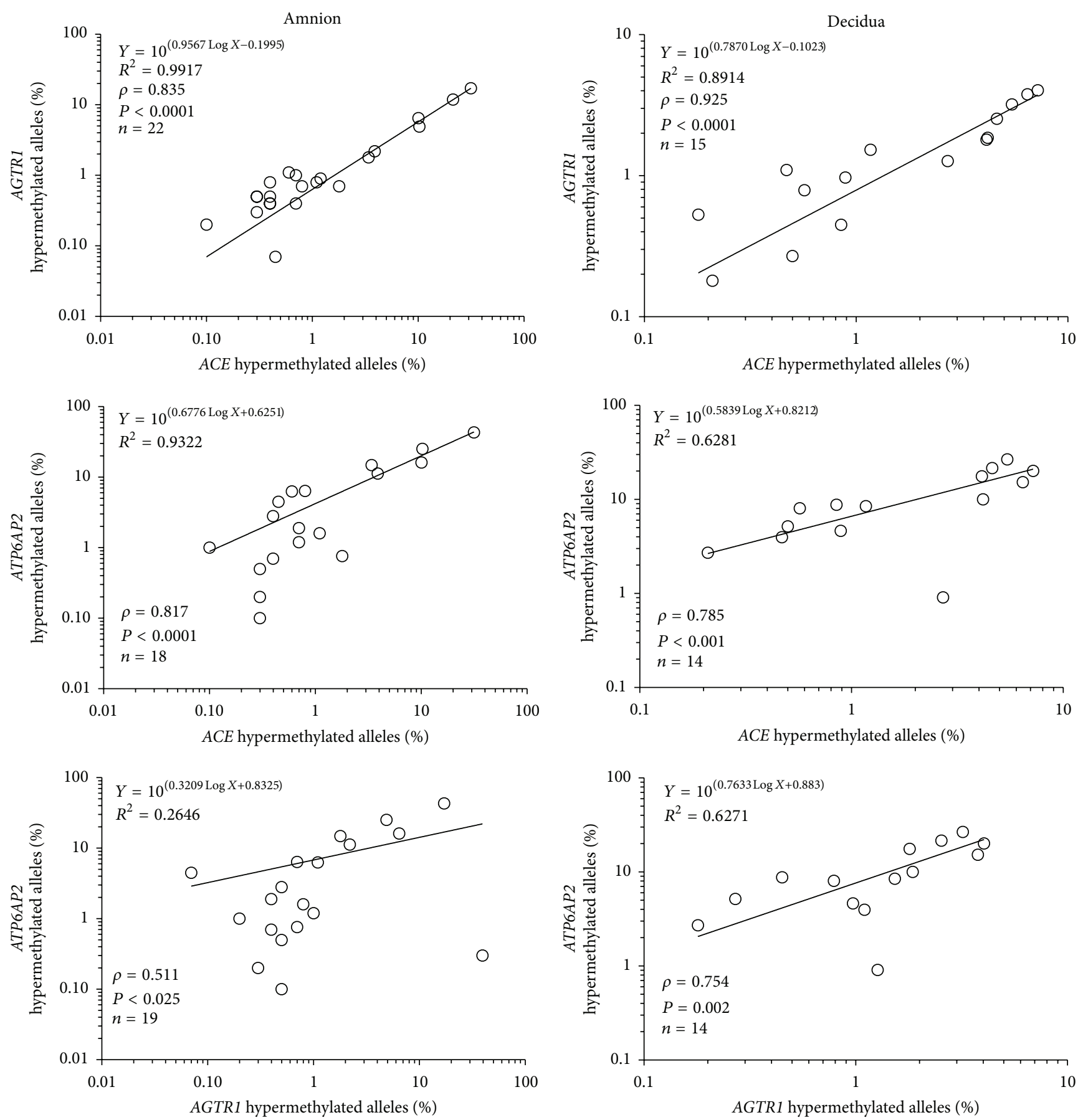

FIGURE 5: Spearman correlations between the proportions of hypermethylated RAS gene alleles within amnion samples isolated from early and late gestation and within decidua samples collected from before and after the onset of labour.

In the placenta, the only correlation found was between the proportions of highly methylated CTSD and KLK1 alleles (rho $=0.964, P<0.003, n=7)$.

To see if the correlated methylation profiles of the 3 RAS genes and CTSD in the decidua and amnion were part of a more widespread gene methylation pattern, we extended the correlation analysis to the proportions of hypermethylated labour associated inflammatory gene alleles (PTGS2, BMP2, NAMPT, and CXCL2) and steroid receptor gene alleles (PGR, ESR1, and NR3C1) in these tissues [24]. We found that hypermethylation of most of these genes correlated with the hypermethylation of RAS and CTSD in the amnion (Table 2). Within the decidua, the hypermethylation of PTGS2, BMP2, CXCL2, and PGR also correlated with the RAS genes (Table 2). The proportion of hypermethylated $K L K 1$ alleles, however, did not correlate with any other gene in either amnion or decidua, including labour associated inflammatory genes and steroid receptor genes. 

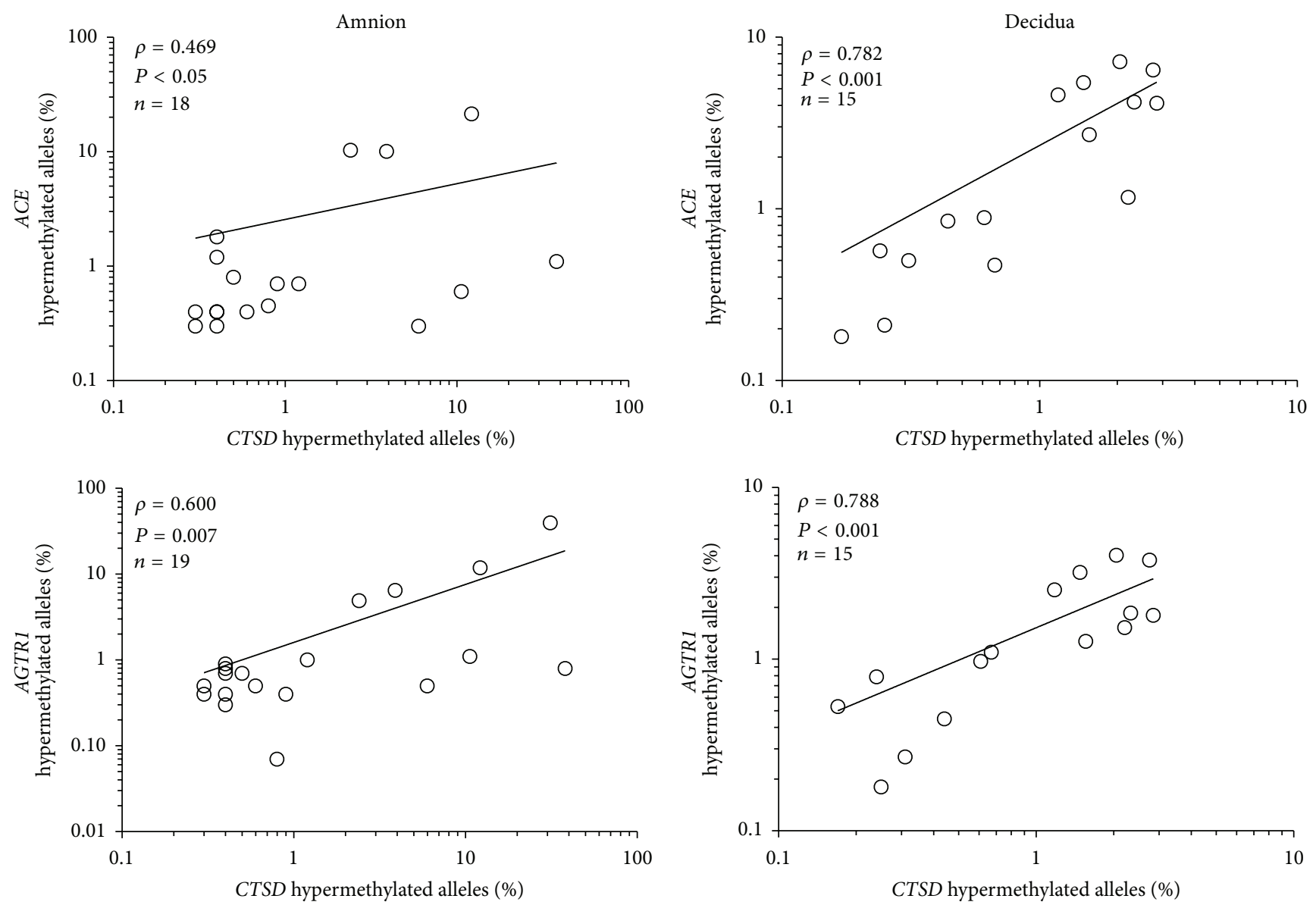

FIGURE 6: Spearman correlations between the proportion of hypermethylated CTSD alleles and the proportion of hypermethylated ACE and AGTR1 alleles in all amnion and decidua samples. ATP6AP2 was not correlated with CTSD.

\section{Discussion}

The principal aim of this project was to explore whether the methylation of RAS and prorenin-activating protease gene promoters influenced the expression of these genes in gestational tissues during pregnancy and labour. We also aimed to assess whether the degree of methylation of these genes were similar to each other, to labour-promoting inflammatory genes, and to genes that encode steroid receptors involved in control of labour [24].

The gestational changes in intrauterine tissue mRNA abundances identified in this study are similar to those that we have previously reported for $A C E, A G T R 1$, and ATP6AP2 [1, 2]. The mRNA abundance of CTSD has not been determined previously in the human amnion. This study demonstrates that in amnion and placenta, tissues of fetal origin, CTSD expression is higher at term. Cathepsin D is a protease involved in trophoblast invasion in the mouse $[25,26]$ and when activated it can cause apoptosis [27], and protein degradation potentially contributing to fetal membrane remodelling at labour.

KLK1 expression has previously been detected in very early gestation human placental tissue and decidua by in situ hybridization, but it has not been measured in amnion
[9, 10]. Apart from activating prorenin, kallikrein can also produce bradykinin, which through the type 2 bradykinin receptor promotes vasodilation, angiogenesis, and invasion in intrauterine tissues [28]. Recently, it has been demonstrated that expression of the type 2 bradykinin receptor is upregulated in decidua and is significantly downregulated in chorionic villous samples from women who develop severe preeclampsia compared to healthy controls [29]. Kallikrein also stimulates the synthesis of labour-promoting PGE in the chorion [30], and drugs that block kallikrein formation delay the onset of labour [31].

There was no relationship in any tissue between the proportions of hypermethylated alleles and the level of expression of the genes. The majority of the alleles were hypomethylated in all tissues, however, indicating that most gene copies were poised for expression under the control of transcriptional regulators.

Early gestation amnion $(n=7)$ had a significantly higher proportion of hypermethylated $K L K 1$ alleles compared with amnion isolated after spontaneous labour and delivery. At the same time, several term amnion samples showed high proportions of intermediately methylated $K L K 1$ alleles, which suggests that $K L K 1$ may be in a state of transition to low methylation density at late gestation. Thus methylation 
TABLE 2: Associations between labour associated inflammatory genes (PTGS2, BMP2, NAMPT, and CXCL2) and steroid receptor genes (PGR, ESR1, and NR3C1) allele hypermethylation and the percentage of gene alleles that were hypermethylated in ACE, AGTR1, ATP6AP2, CTSD, and $K L K 1$ in amnion and decidua.

\begin{tabular}{|c|c|c|c|c|c|}
\hline & ACE1 & AGTR1 & ATP6AP2 & CTSD & $K L K 1$ \\
\hline \multicolumn{6}{|l|}{ Amnion } \\
\hline \multirow{2}{*}{ PTGS2 } & $r=0.85$ & $r=0.81$ & $r=0.50$ & $r=0.60$ & \multirow{2}{*}{ ns } \\
\hline & $P<0.001$ & $P<0.001$ & $P=0.036$ & $P<0.01$ & \\
\hline \multirow{2}{*}{$B M P 2$} & $r=0.83$ & $r=0.90$ & $r=0.57$ & $r=0.82$ & \multirow{2}{*}{ ns } \\
\hline & $P<0.001$ & $P<0.001$ & $P=0.017$ & $P<0.001$ & \\
\hline \multirow{2}{*}{ NAMPT } & $r=0.71$ & $r=0.72$ & \multirow{2}{*}{ ns } & $r=0.68$ & \multirow{2}{*}{ ns } \\
\hline & $P<0.001$ & $P<0.001$ & & $P=0.001$ & \\
\hline \multirow{2}{*}{ CXCL2 } & $r=0.94$ & $r=0.80$ & $r=0.69$ & $r=0.56$ & \multirow{2}{*}{ ns } \\
\hline & $P<0.001$ & $P<0.001$ & $P=0.002$ & $P=0.013$ & \\
\hline \multirow{2}{*}{$N R 3 C 1$} & $r=0.72$ & $r=0.78$ & $r=0.49$ & $r=0.49$ & \multirow{2}{*}{ ns } \\
\hline & $P<0.001$ & $P<0.001$ & $P=0.035$ & $P=0.03$ & \\
\hline \multirow{2}{*}{$P G R$} & $r=0.85$ & $r=0.83$ & $r=0.53$ & $r=0.71$ & \multirow{2}{*}{ ns } \\
\hline & $P<0.001$ & $P<0.001$ & $P=0.02$ & $P<0.001$ & \\
\hline \multirow{2}{*}{ ESR1 } & $r=0.87$ & $r=0.77$ & $r=0.71$ & $r=0.49$ & \multirow{2}{*}{ ns } \\
\hline & $P<0.001$ & $P<0.001$ & $P=0.001$ & $P=0.038$ & \\
\hline \multicolumn{6}{|l|}{ Decidua } \\
\hline \multirow{2}{*}{ PTGS2 } & $r=0.75$ & $r=0.72$ & \multirow{2}{*}{ ns } & $r=0.86$ & \multirow{2}{*}{ ns } \\
\hline & $P=0.001$ & $P=0.003$ & & $P<0.001$ & \\
\hline \multirow{2}{*}{$B M P 2$} & $r=0.90$ & $r=0.90$ & $r=0.62$ & $r=0.84$ & \multirow{2}{*}{ ns } \\
\hline & $P<0.001$ & $P<0.001$ & $P=0.033$ & $P<0.001$ & \\
\hline \multirow{2}{*}{ NAMPT } & $r=0.55$ & & $\mathrm{~ns}$ & $r=0.83$ & \multirow{2}{*}{ ns } \\
\hline & $P=0.04$ & ns & ns & $P<0.001$ & \\
\hline \multirow{2}{*}{ CXCL2 } & $r=0.88$ & $r=0.82$ & $r=0.78$ & $r=0.77$ & \multirow{2}{*}{ ns } \\
\hline & $P<0.001$ & $P<0.001$ & $P=0.001$ & $P=0.001$ & \\
\hline NR3C1 & ns & ns & ns & ns & ns \\
\hline \multirow{2}{*}{$P G R$} & $r=0.93$ & $r=0.94$ & $r=0.71$ & $r=0.87$ & \multirow{2}{*}{ ns } \\
\hline & $P<0.001$ & $P<0.001$ & $P=0.005$ & $P<0.001$ & \\
\hline \multirow{2}{*}{ ESR1 } & $r=0.72$ & $r=0.80$ & \multirow{2}{*}{ ns } & $r=0.83$ & \multirow{2}{*}{ ns } \\
\hline & $P=0.003$ & $P<0.001$ & & $P<0.001$ & \\
\hline
\end{tabular}

ns = not significant

appears dynamic in particular gene(s) even at term, although the impact of this methylation change on $K L K 1$ gene activity remains unclear.

The wide ranging variation in methylation of RAS genes between individuals, the strong correlations between the proportions of hypermethylated alleles of the 3 RAS genes and CTSD in amnion and decidua, and the correlations between methylation profiles of these 4 genes and labour associated gene alleles and steroid receptor gene alleles [32] in amnion suggest that there is a global influence on DNA methylation in the gestational tissues affecting numerous genes. The level of DNA methylation of these genes is set possibly by individual conditions early in gestation [33].

In placentas, high proportions of intermediately methylated alleles were found for several genes (AGTR1, ATP6AP2, $C T S D$, and KLK1) at early gestation ( $<18$ weeks). In contrast, none of the placental samples collected at term in the absence of labour (>37 weeks of gestation) contained detectable levels of intermediately methylated alleles of these genes, however, and the promoters were predominantly hypomethylated. This observation is consistent with the view that DNA methylation is most dynamic in early pregnancy and in the placenta low global methylation levels are established [12].

In conclusion, the promoter methylation status of the studied RAS and renin-activating protease genes has no apparent influence on either the gestational or the labour associated changes of expression in intrauterine tissues reported in previous studies $[1,34]$. Promoter methylation is dynamic in the placenta at early pregnancy and in the case of one gene (KLK1) in late gestation amnion. The majority of the allele populations are unmethylated, however, and these gene copies may be subject to transcriptional regulation. Future studies focusing on histone modifications may reveal epigenetic mechanisms that have an impact on gene activity in the context of placental function, pregnancy maintenance, and parturition. 


\section{Ethical Approval}

The authors assert that all procedures contributing to this work comply with the ethical standards of the relevant national guidelines on human experimentation (National Statement on Ethical Conduct in Human Research, 2007, Australia) and with the Helsinki Declaration of 1975, as revised in 2008, and the work has been approved by the institutional committees (Hunter Area Research Ethics Committee and the University of Newcastle Research Ethics Committee).

\section{Conflict of Interests}

The authors declare that there is no conflict of interests regarding the publication of this paper.

\section{Acknowledgments}

The authors would like to thank David Mossman for kindly supplying the cDNA from SW48 cells used as the calibrator for KLK1 gene expression. This work was supported by the National Health and Medical Research Council, Australia (Grant no. 510746).

\section{References}

[1] K. G. Pringle, M. A. Tadros, R. J. Callister, and E. R. Lumbers, "The expression and localization of the human placental prorenin/renin-angiotensin system throughout pregnancy: roles in trophoblast invasion and angiogenesis?" Placenta, vol. 32, no. 12, pp. 956-962, 2011.

[2] F. Z. Marques, K. G. Pringle, A. Conquest et al., "Molecular characterization of renin-angiotensin system components in human intrauterine tissues and fetal membranes from vaginal delivery and cesarean section," Placenta, vol. 32, no. 3, pp. 214221, 2011.

[3] G. Nguyen, F. Delarue, C. Burcklé, L. Bouzhir, T. Giller, and J.-D. Sraer, "Pivotal role of the renin/prorenin receptor in angiotensin II production and cellular responses to renin," Journal of Clinical Investigation, vol. 109, no. 11, pp. 1417-1427, 2002.

[4] R. Haddad, G. Tromp, H. Kuivaniemi et al., "Human spontaneous labor without histologic chorioamnionitis is characterized by an acute inflammation gene expression signature," The American Journal of Obstetrics and Gynecology, vol. 195, no. 2, pp. 394-e12, 2006.

[5] A. R. Brasier, A. Recinos III, and M. S. Eledrisi, "Vascular inflammation and the renin-angiotensin system," Arteriosclerosis, Thrombosis, and Vascular Biology, vol. 22, no. 8, pp. 12571266, 2002.

[6] S. Lundin-Schiller and M. D. Mitchell, "Renin increases human amnion cell prostaglandin E2 biosynthesis," Journal of Clinical Endocrinology and Metabolism, vol. 73, no. 2, pp. 436-440, 1991.

[7] U. Earl, L. Morrison, C. Gray, and J. N. Bulmer, "Proteinase and proteinase inhibitor localization in the human placenta," International Journal of Gynecological Pathology, vol. 8, no. 2, pp. 114-124, 1989.

[8] A. Bergqvist, M. Ferno, and S. Mattson, "A comparison of cathepsin D levels in endometriotic tissue and in uterine endometrium," Fertility and Sterility, vol. 65, no. 6, pp. 1130-1134, 1996.
[9] G. Valdés, C. Chacón, J. Corthorn, C. D. Figueroa, and A. M. Germain, "Tissue kallikrein in human placenta in early and late gestation," Endocrine, vol. 14, no. 2, pp. 197-204, 2001.

[10] G. Valdés, A. M. Germain, J. Corthorn, C. Chacón, C. D. Figueroa, and W. Müller-Esterl, "Tissue kallikrein and bradykinin B2 receptor in human uterus in luteal phase and in early and late gestation," Endocrine, vol. 16, no. 3, pp. 207-215, 2001.

[11] J. B. Su, "Different cross-talk sites between the renin-angiotensin and the kallikrein-kinin systems," Journal of the ReninAngiotensin-Aldosterone System, vol. 15, no. 4, pp. 319-328, 2014.

[12] E. C. M. Nelissen, A. P. A. van Montfoort, J. C. M. Dumoulin, and J. L. H. Evers, "Epigenetics and the placenta," Human Reproduction Update, vol. 17, no. 3, pp. 397-417, 2011.

[13] J. Rossant, J. P. Sanford, V. M. Chapman, and G. K. Andrews, "Undermethylation of structural gene sequences in extraembryonic lineages of the mouse," Developmental Biology, vol. 117, no. 2, pp. 567-573, 1986.

[14] W. Dean, F. Santos, M. Stojkovic et al., "Conservation of methylation reprogramming in mammalian development: aberrant reprogramming in cloned embryos," Proceedings of the National Academy of Sciences of the United States of America, vol. 98, no. 24, pp. 13734-13738, 2001.

[15] H.-M. Byun, K. D. Siegmund, F. Pan et al., "Epigenetic profiling of somatic tissues from human autopsy specimens identifies tissue- and individual-specific DNA methylation patterns," Human Molecular Genetics, vol. 18, no. 24, pp. 4808-4817, 2009.

[16] B. C. Christensen, E. A. Houseman, C. J. Marsit et al., "Aging and environmental exposures alter tissue-specific DNA methylation dependent upon CPG island context," PLoS Genetics, vol. 5, no. 8, Article ID e1000602, 2009.

[17] G. Nagae, T. Isagawa, N. Shiraki et al., "Tissue-specific demethylation in CpG-poor promoters during cellular differentiation," Human Molecular Genetics, vol. 20, no. 14, pp. 2710-2721, 2011.

[18] K. G. Pringle, T. Zakar, D. Yates, C. M. Mitchell, J. J. Hirst, and E. R. Lumbers, "Molecular evidence of a (pro)renin/ (pro)renin receptor system in human intrauterine tissues in pregnancy and its association with PGHS-2," Journal of the Renin-AngiotensinAldosterone System, vol. 12, no. 3, pp. 304-310, 2011.

[19] R. F. Johnson, C. M. Mitchell, V. Clifton, and T. Zakar, "Regulation of 15-hydroxyprostaglandin dehydrogenase (PGDH) gene activity, messenger ribonucleic acid processing, and protein abundance in the human chorion in late gestation and labor," Journal of Clinical Endocrinology and Metabolism, vol. 89, no. 11, pp. 5639-5648, 2004.

[20] H. Holemon, Y. Korshunova, J. M. Ordway et al., "MethylScreen: DNA methylation density monitoring using quantitative PCR," BioTechniques, vol. 43, no. 5, pp. 683-693, 2007.

[21] J. M. Ordway, J. A. Bedell, R. W. Citek et al., "Comprehensive DNA methylation profiling in a human cancer genome identifies novel epigenetic targets," Carcinogenesis, vol. 27, no. 12, pp. 2409-2423, 2006.

[22] T. J. Harvey, J. D. Hooper, S. A. Myers, S.-A. Stephenson, L. K. Ashworth, and J. A. Clements, "Tissue-specific expression patterns and fine mapping of the human kallikrein (KLK) locus on proximal 19q13.4," The Journal of Biological Chemistry, vol. 275, no. 48, pp. 37397-37406, 2000.

[23] K. J. Livak and T. D. Schmittgen, "Analysis of relative gene expression data using real-time quantitative PCR and the 2[delta][delta]CT method," Methods, vol. 25, no. 4, pp. 402-408, 2001.

[24] C. M. Mitchell, S. D. Sykes, X. Pan et al., "Inflammatory and steroid receptor gene methylation in the human amnion and 
decidua," Journal of Molecular Endocrinology, vol. 50, no. 2, pp. 267-277, 2013.

[25] A. M. Amarante-Paffaro, M. S. Hoshida, S. Yokota et al., "Localization of cathepsins D and B at the maternal-fetal interface and the invasiveness of the trophoblast during the postimplantation period in the mouse," Cells Tissues Organs, vol. 193, no. 6, pp. 417-425, 2011.

[26] S. Afonso, L. Romagnano, and B. Babiarz, "Expression of cathepsin proteinases by mouse trophoblast in vivo and in vitro," Developmental Dynamics, vol. 216, no. 4-5, pp. 374-384, 1999.

[27] Y. Gan, X. Zhao, J. Hu, Z. G. Wang, and X. T. Zhao, "HCCS1 overexpression induces apoptosis via cathepsin D and intracellular calcium, and HCCS1 disruption in mice causes placental abnormality," Cell Death and Differentiation, vol. 15, no. 9, pp. 1481-1490, 2008.

[28] G. Valdés and J. Corthorn, "Review: the angiogenic and vasodilatory utero-placental network," Placenta, vol. 32, supplement 2, pp. S170-S175, 2011.

[29] M. B. Rabaglino, E. D. Post Uiterweer, A. Jeyabalan, W. A. Hogge, and K. P. Conrad, "Bioinformatics approach reveals evidence for impaired endometrial maturation before and during early pregnancy in women who developed preeclampsia," Hypertension, vol. 65, no. 2, pp. 421-429, 2015.

[30] G. M. Acker and A. Pesty, "Effects of fetal urinary corticosteroids, catecholamines and kallikrein on PGE2 synthesis in monolayer cultures of human amnion and chorion cells," Prostaglandins, Leukotrienes and Essential Fatty Acids, vol. 34, no. 3, pp. 135-140, 1988.

[31] J. Senior and E. T. Whalley, “The influence of drugs on the kinin forming system in relation to pregnancy and parturition in the rat," Journal of Reproduction and Fertility, vol. 47, no. 2, pp. 319323, 1976.

[32] C. Mitchell, R. Johnson, A. Bisits, J. Hirst, and T. Zakar, "PTGS2 (prostaglandin endoperoxide synthase-2) expression in term human amnion in vivo involves rapid mRNA turnover, polymerase-II $5^{\prime}$-pausing, and glucocorticoid transrepression," Endocrinology, vol. 152, no. 5, pp. 2113-2122, 2011.

[33] B. Novakovic and R. Saffery, "The ever growing complexity of placental epigenetics - role in adverse pregnancy outcomes and fetal programming," Placenta, vol. 33, no. 12, pp. 959-970, 2012.

[34] Y. Wang, K. G. Pringle, S. D. Sykes et al., "Fetal sex affects expression of renin-angiotensin system components in term human decidua," Endocrinology, vol. 153, no. 1, pp. 462-468, 2012. 


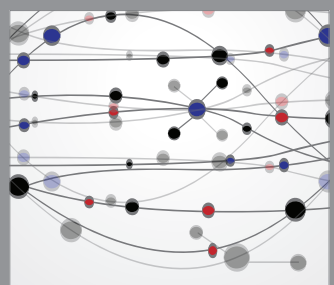

The Scientific World Journal
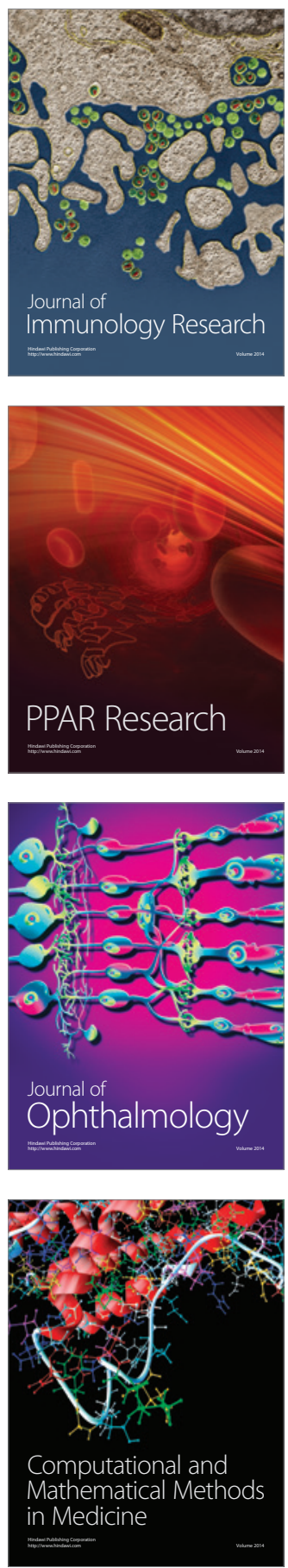

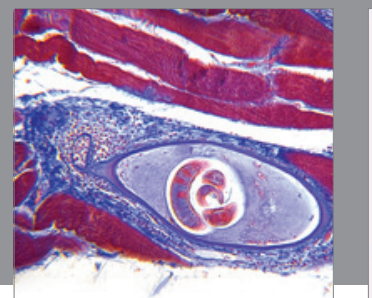

Gastroenterology

Research and Practice
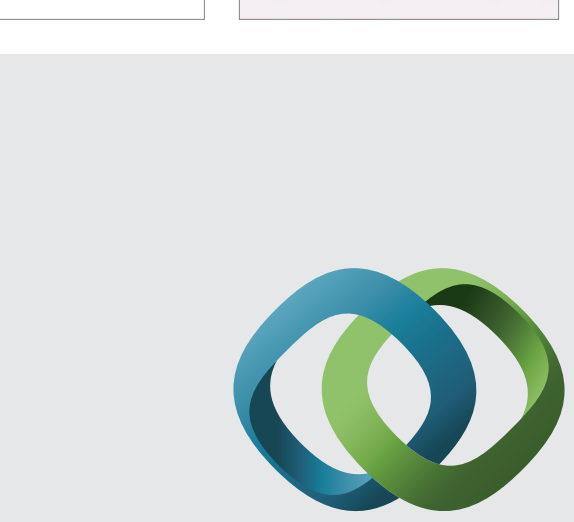

\section{Hindawi}

Submit your manuscripts at

http://www.hindawi.com
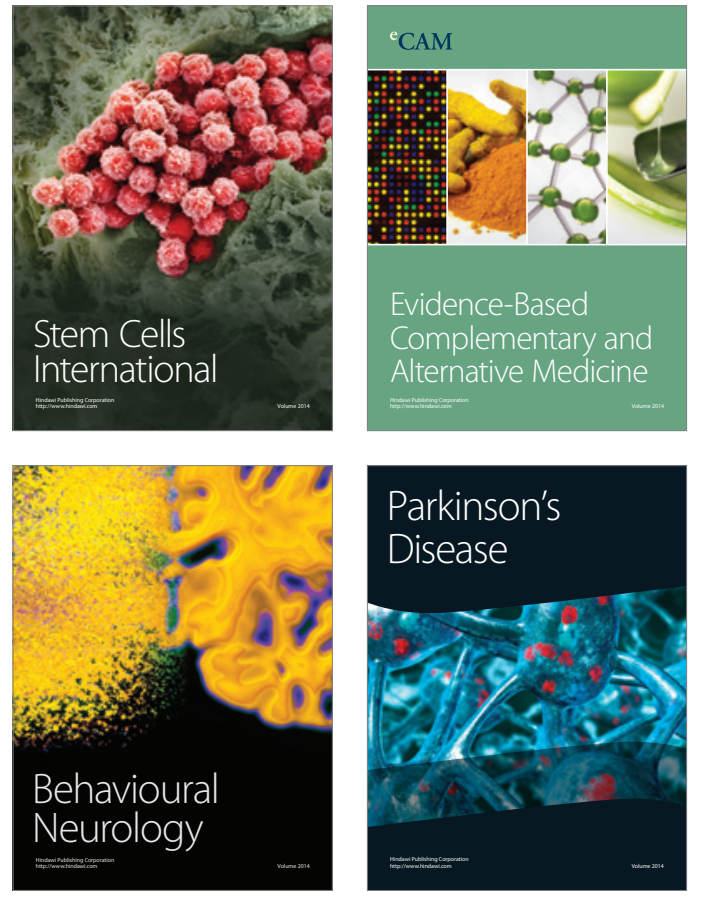
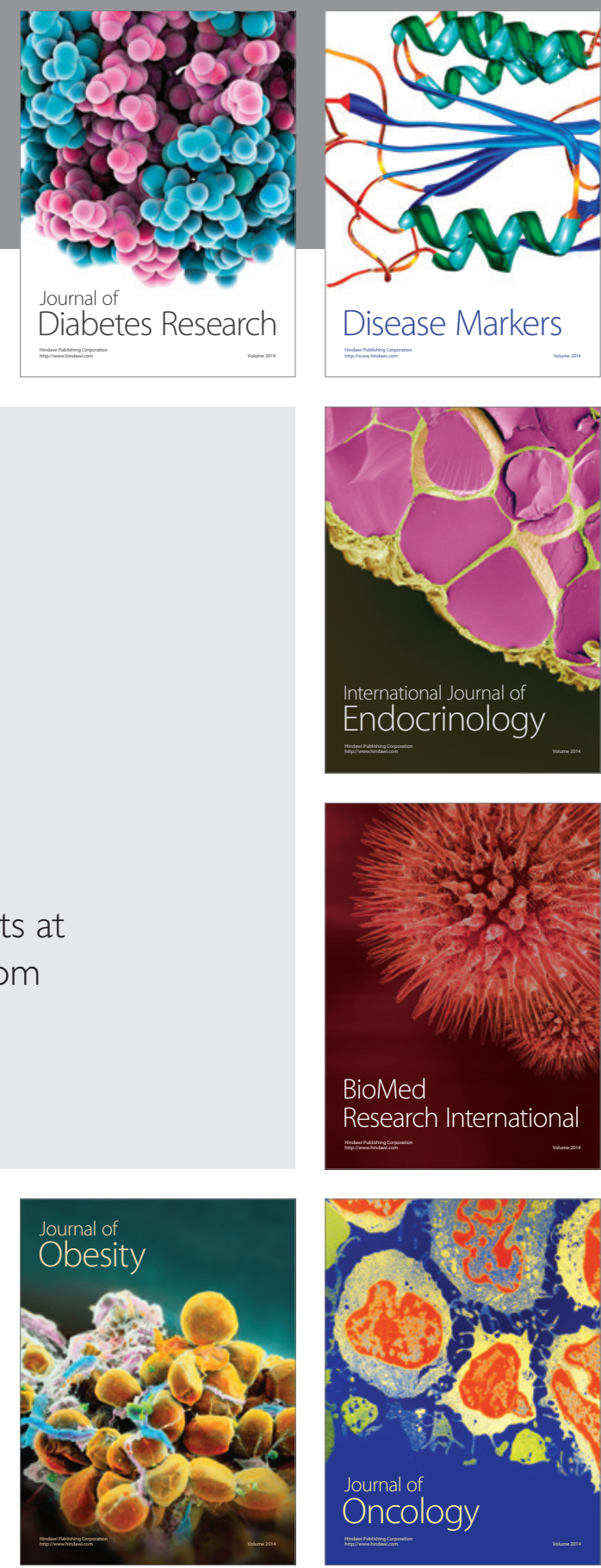

Disease Markers
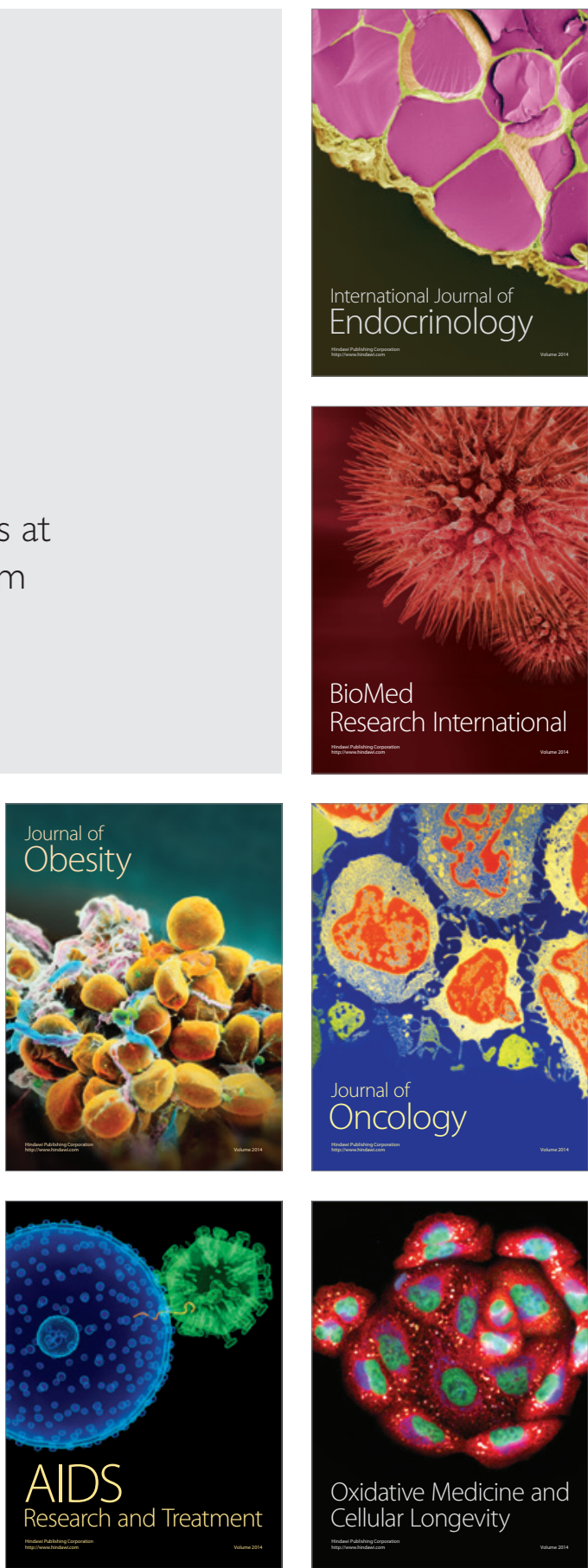